\title{
HOMOGENEOUS ALMOST TANGENT STRUCTURES
}

\section{P. CLOSS}

1. Introduction. Let $J$ be a field of linear operators acting on the complexified tangent spaces of a differentiable manifold $V_{2 n}$, of dimension $2 n$, and which satisfies a relation of the form $J^{2}=\lambda^{2} I$ where $\lambda$ is a complex constant and $I$ is the identity operator. In the case $\lambda \neq 0$ the manifold has an almost product structure which in the case $\lambda=i$ reduces to an almost complex structure. In the remaining case, $\lambda=0$, (rank $J=n)$ the manifold has an almost tangent structure [1]. In [2] Frolicher has studied homogeneous almost complex structures and in [3] Legrand has studied homogeneous almost product structures. In the present paper the author extends this treatment to the almost tangent structures.

2. Notation. Let $G$ be a connected Lie group and $H$ a closed subgroup. The quotient space $G / H$ (under the equivalence relation $\left.g_{1}^{-1} g_{2} \in H\right)$ is able to be endowed with the structure of a real analytic manifold $V_{m}$ with an analytic projection $p: G \rightarrow G / H$. $G$ has with respect to $p$, a natural structure of a principal fibre bundle with base space $V_{m}$ and structural group $H$ effective on itself by left translation.

Let us be given an element $\xi \in G$. To the point $x=p g$ (where $g \in G$ ) of $V_{m}$, let us correspond the point

$$
K_{\xi} x=p(\xi g) .
$$

$K_{\xi} x$ is independent of the choice of $g$ in $p^{-1}(x) . G$ thus appears as a transitive group of analytic transformations of $V_{m}$. We will say that $V_{m}$, furnished with the structure defined by the group of operators $G$, is a homogeneous Lie space.

Let the differential of $K_{\xi}$ at $x$ be denoted by $K_{\xi}^{\prime}(x)$. Then $K_{\xi}^{\prime}(x)$ is a linear mapping of the tangent vector space at $x$ onto the tangent vector space at $K_{\xi} x$. In particular at the point $x_{0}=p e$ (where $e$ is the identity of $G$ ) and for $h \in H$ we have $K_{h}^{\prime}\left(x_{0}\right)$ is a linear mapping of $T_{x_{0}}$ into itself. We may thus represent $H$ by a group $\tilde{H}$ of linear transformations of $T_{x_{0}}$. We shall briefly denote $K_{\xi}^{\prime}\left(x_{0}\right)$ by $K_{\xi}^{\prime}$.

If $p^{\prime}: T_{0} \rightarrow T_{p o}$ denotes the linear mapping of the tangent vector spaces of $G$ onto the tangent vector spaces of $V_{m}$ we will have for any $u \in T_{e}$

$$
K_{h}^{\prime} p^{\prime} u=p^{\prime}(\operatorname{adj} h) u
$$

Received by the editors November 15, 1968 and, in revised form, April 9, 1969. 
where $(\operatorname{adj} h)$ is the element corresponding to $h \in H$ under the adjoint representation of $G$.

The extension of the mapping $K_{\xi}^{\prime}(x)$ to the tensors defined on $T_{x}$ will be represented by the same symbol. In particular, for the case of a tensor $J$ of type $(1,1)$ defined on $T_{x_{0}}$ we will have

$$
K_{\xi}^{\prime}(J)=K_{\xi-1}^{\prime} \circ J \circ K_{\xi}^{\prime} \text {. }
$$

We will assume in the sequel that $V_{m}$ is of dimension $m=2 n$ for some $n$.

3. Homogeneous almost tangent structures. Let us consider on a homogeneous Lie space $V_{2 n}=G / H$ a field of real or complex tensors; let $t_{x}$ be the tensor of the field at the point $x=p g$. A necessary and sufficient condition that the field be invariant under $G$ is that

$$
t_{x}=K_{a}^{\prime}\left(t_{x_{0}}\right)
$$

for an arbitrary $g \in p^{-1}\left(x_{0}\right)$, that is, be sent into itself by each element of the group $G$. Clearly, $t_{x_{0}}$ is invariant under $\tilde{H}$. From a tensor defined at the point $x_{0}$ and invariant under $\widetilde{H}$, we may use the formula (1) to define on $V_{m}$ a field of tensors invariant under $G$.

In particular let us suppose that there exists at the point $x_{0}$ a tensor $\left(F_{i}^{s}\right)$, invariant under $\widetilde{H}$ and of rank $n$ such that

$$
F_{i}^{j} F_{j}^{k}=0 .
$$

One deduces from this by the formula (1) a field of tensors invariant under $G$ and which satisfies the relation (2) at each point of $V_{2 n}$. It thus determines an almost tangent structure on $V_{2 n}$. We will then say that $V_{2 n}$ is furnished with a homogeneous almost tangent structure.

If $J$ is a $(1,1)$ tensor defined on $T_{x_{0}}$ then it will be invariant under $H$ if and only if

$$
K_{h}^{\prime}(J)=J
$$

that is

$$
K_{h}^{\prime}-1 \circ J \circ K_{h}^{\prime}=J
$$

or

$$
J \circ K_{h}^{\prime}=K_{h}^{\prime} \circ J \quad \text { for any } h \in H .
$$

$T_{x_{0}}^{c}$ being the complexified vector space of $T_{x_{0}}$, a homogeneous almost tangent structure is able to be defined by the data on $T_{x_{0}}^{c}$ of a linear operator $J$, of rank $n$, satisfying the following conditions: 


$$
\begin{gathered}
J^{2}=0 \\
J \circ K_{h}^{\prime}=K_{h}^{\prime} \circ J \quad \text { for any } h \in H .
\end{gathered}
$$

(3) is the translation of (2) in terms of operators and (4) expresses the invariance of $J$ under $\widetilde{H}$.

Let us denote by $G$ the tangent vector space to $G$ at the point $e$, furnished with its Lie algebra structure; by $G^{c}$ its complexification; and by $H$ and $H^{c}$ the analogous spaces relative to the subgroup $H$ of $G$. Let us choose in $G^{c}$ a subspace $M$ supplementary to $H^{c}$, that is such that $G^{c}=H^{c} \oplus M$. Since any $R$-linear map between real vector spaces extends in a unique way to a $C$-linear map of the complexified spaces, the mapping $p^{\prime}: G \rightarrow T_{x_{0}}$ is able to be extended to $G^{c}$. By restriction to $M$ it determines an isomorphism of $M$ onto $T_{x_{0}}^{c}$, for which we denote the inverse isomorphism by $q^{\prime}$.

To any linear operator $J$ defined on $T_{x_{0}}^{c}$ one is able to associate the linear operator $I: G^{c} \rightarrow G^{c}$ defined by setting $I u=q^{\prime} J p^{\prime} u$ for any $u \in G^{c}$.

If $J$ is of rank $n$ on $T_{x_{0}}^{c}$, then $J\left(T_{x_{0}}^{c}\right)$ is of dimension $n$ and since $q^{\prime}$ is an isomorphism of $T_{x_{0}}^{c}$ onto $M$ then $I\left(G^{c}\right)$ is an $n$-dimensional subspace of $M$. Thus $I$ is a linear operator of rank $n$, zero on $H^{c}$, and is such that $I\left(G^{c}\right) \cap H^{c}=O$. We note that $p^{\prime} q^{\prime}$ is the identity on $J\left(T_{x}^{c *}\right)$ and $q^{\prime} p^{\prime}$ is the identity on $M$.

Let $h \in H$; one then has for any $u \in G^{c}$

$$
\begin{aligned}
p^{\prime}(\operatorname{adj} h) I u & =K_{h}^{\prime} p^{\prime} I u \\
& =K_{h}^{\prime} p^{\prime} q^{\prime} J p^{\prime} u \\
& =K_{h}^{\prime} J p^{\prime} u
\end{aligned}
$$

and

$$
\begin{aligned}
p^{\prime} I(\operatorname{adj} h) u & =p^{\prime} q^{\prime} J p^{\prime}(\operatorname{adj} h) u \\
& =J p^{\prime}(\operatorname{adj} h) u \\
& =J K_{h}^{\prime} p^{\prime} u
\end{aligned}
$$

Hence

$$
p^{\prime}((\operatorname{adj} h) I-I(\operatorname{adj} h)) u=\left(K_{h}^{\prime} J-J K_{h}^{\prime}\right) p^{\prime} u .
$$

Suppose now that $J$ satisfies (3) and (4); then for any $u \in G^{c}$ the operator $I$ associated to $J$ is such that:

$$
I^{2} u=q^{\prime} J p^{\prime} q^{\prime} J p^{\prime} u=q^{\prime} J^{2} p^{\prime} u=0
$$

and according to (5) such that: 


$$
p^{\prime}((\operatorname{adj} h) I-I(\operatorname{adj} h)) u=0
$$

for any $h \in H$. One is then able to write

$$
I^{2} u=0
$$

$$
(\operatorname{adj} h) I u \equiv I(\operatorname{adj} h) u \quad\left(\operatorname{modulo} H^{c}\right)
$$

for any $u \in G^{c}$ and $h \in H$.

Conversely, let $I$ be a linear operator of $G^{c}$ into itself such that $I\left(G^{c}\right) \cap H^{c}=O$ where $I$ is zero on $H^{c}$, of rank $n$, and satisfies (6) and (7). Then $I\left(G^{c}\right)$ is an $n$-dimensional subspace of $M$ where $M$ is supplementary to $H^{c}$ in $G^{c}$. Let $q^{\prime}$ be, as earlier, the isomorphism of $T_{x_{0}}^{c}$ onto $M$. We define a linear operator $J$ of $T_{x_{0}}^{c}$ into itself by setting $J v=p^{\prime} I q^{\prime} v$ for any $v \in T_{x_{0}}^{c}$. Thus $J$ is of rank $n$. It also follows that

$$
J^{2} v=p^{\prime} I q^{\prime} p^{\prime} I q^{\prime} v=p^{\prime} I^{2} q^{\prime} v=0
$$

for any $v \in T_{x_{0}}^{c}$.

Let us put $u=u_{H}+u_{M}$ where $u_{H} \in H^{c}, u_{M} \in M$, for any $u \in G^{c}$. We then have $g^{\prime} v=u_{M}$ whenever $p^{\prime} u=v$. Now,

$$
\begin{aligned}
K_{h}^{\prime} J v & =K_{h}^{\prime} p^{\prime} I q^{\prime} v \\
& =p^{\prime}(\operatorname{adj} h) I q^{\prime} v \\
& =p^{\prime}(\operatorname{adj} h) I u
\end{aligned}
$$

where $p^{\prime} u=v$ since $I u=I\left(u_{H}+u_{M}\right)=I u_{M}=I q^{\prime} v$. In addition we have

$$
\begin{aligned}
J K_{h}^{\prime} v & =p^{\prime} I q^{\prime} K_{h}^{\prime} p^{\prime} u \quad \text { where } p^{\prime} u=v \\
& =p^{\prime} I q^{\prime} p^{\prime}(\operatorname{adj} h) u \\
& =p^{\prime} I q^{\prime} p^{\prime}\left(w_{H}+w_{M}\right) \quad \text { where }(\operatorname{adj} h) u=w \\
& =p^{\prime} I q^{\prime} p^{\prime} w_{M} \\
& =p^{\prime} I w_{M} \\
& =p^{\prime} I\left(w_{H}+w_{M}\right) \\
& =p^{\prime} I(\operatorname{adj} h) u .
\end{aligned}
$$

Hence for any $v \in T_{x_{0}}^{c}$ and any $h \in H$ we have

$$
\begin{aligned}
\left(K_{h}^{\prime} J-J K_{h}^{\prime}\right) v & =p^{\prime}((\operatorname{adj} h) I-I(\operatorname{adj} h)) u \\
& =0
\end{aligned}
$$

where $p^{\prime} u=v$.

We have thus proven

Theorem 1. In order that a homogeneous lie space $V_{2 n}=G / H$ be able to be endowed with a homogeneous almost tangent structure it is 
necessary and sufficient that there exists on $G^{c}$ a linear operator I satisfying the following conditions:

(a) I is zero on $H^{c}$,

(b) rank $I=n$,

(c) $I\left(G^{c}\right) \cap H^{c}=O$,

(d) $I^{2} u=0$ for any $u \in G^{c}$,

(e) $(\operatorname{adj} h) I u \equiv I(\operatorname{adj} h) u \quad\left(\bmod H^{c}\right)$ for any $u \in G^{c}$ and $h \in H$.

If (7) is satisfied for any $h \in H$, it will in particular be satisfied for the elements $h=\exp (t z)$ of the one parameter subgroup generated by an element $z$ of $\boldsymbol{H}$. By passage to the Lie algebras one deduces from this that the condition (7) leads to

$$
[z, I u] \equiv I[z, u] \quad\left(\bmod H^{c}\right)
$$

for any $u \in G^{c}$ and $z \in H$. $I$ being a linear operator on $G^{c}$, this relation extends to the elements $z \in H^{c}$.

Conversely, if (8) is satisfied, the condition (7) is satisfied for the elements $h \in H$ such that (adj $h$ ) belongs to a one parameter subgroup generated by an element of the Lie algebra of the group $(\operatorname{adj} H)$. If this group is connected, (7) is then satisfied for arbitrary $h$.

Theorem 2. In order that a homogeneous Lie space $V_{2 n}=G / H$ be able to be endowed with a homogeneous almost tangent structure, it is necessary that there exists on $G^{c}$ a linear operator I satisfying the following conditions:

(a) $I$ is zero on $H^{c}$,

(b) $\operatorname{rank} I=n$,

(c) $I\left(G^{c}\right) \cap H^{c}=0$,

(d) $I^{2} u=0$ for any $u \in G^{c}$,

(e) $\left[I u, u_{H}^{\prime}\right] \equiv I\left[u, u_{H}^{\prime}\right]\left(\bmod H^{c}\right)$ for any $u \in G^{c}$ and $u_{H}^{\prime} \in H^{c}$.

These conditions are sufficient if $(\operatorname{adj} H)$ is connected.

\section{REFERENCES}

1. H. A. Eliopoulos, On the general theory of differentiable manifolds with almost tangent structure, Canad. Math. Bull. 8 (1965), 721-748.

2. A. Frolicher, Zur differentialgeometrie der komplexen strukturen, Math. Ann. 129 (1955), 50-95.

3. G. Legrand, Etude d'une généralization des structures presque complexes sur les variêtés differentiables, Rend. Circ. Mat. Palermo (2), 7 (1958), 323-354; ibid. 8 (1959), 5-48.

UNIVERSITY OF OTTAWA 